\title{
Effect of Thermal Sensor Calibration on PCR Chip Performance
}

\author{
Seul-Bit-Na Koo, Jae-Hyeon Cho, Yu-Seop Kim, Hye-Jeong Song, Chan-Young \\ Park and Jong-Dae Kim* \\ Department of Convergence Software, Hallym, Chunchon, Korea \\ Bio-IT Research Center, Hallym, Chunchon, Korea \\ \{rntmfqlcsk, jhcho1028, hjsong, yskim01,cypark, kimjd\}@hallym.ac.kr
}

\begin{abstract}
The fabrication of microfluidic channels in previously proposed polymerase chain reaction (PCR) chips by using polydimethylsiloxane, polycarbonate, and poly(methyl methacrylate) is expensive. To reduce the cost of the disposable chips, a polymer film is employed on the polychlorinated biphenyl (PCB) substrate for the bottom, a carved double-sided tape is used for the wall, and a film with inlet and outlet holes is used for the ceiling of the reaction chamber. The chip temperature is controlled by the heater pattern of the substrate and the thermistor on the opposite face. Commercial chip thermistors with a $1 \%$ resistance tolerance are adapted. In this paper, the effect of the thermistor calibration on PCR performance is investigated. A comparison ofresults on the band brightness of gel images indicated that the calibration seemed to deliver abetter performance.
\end{abstract}

Keywords:PCR, Micro-PCR chip, calibration, thermistor

\section{Introduction}

A polymerase chain reaction (PCR) test has advantages in that the test can be conducted with a small amount of DNA and is used in the diagnosis of various diseases. However, the current commercialized PCR devices have several weaknesses: the test takes a long time, moving the devices is difficult owing to their size, and the devices are expensive [1]. Now that lab-on-a-chip systems are being used for DNA analysis, the development of a PCR chip that has amicrofluidic channel is being carried out to overcome the previous weaknesses [1-3]. In aPCR chip, by using a very small amount of DNA, various data about the gene can be obtained quickly, and the gene can be analyzed. The PCR chip is used to diagnose various diseases through a test subject's reaction with its internal component [3]. To commercialize this capability, the PCR chip must be reliable, reproducible, and sensitive. Therefore, accurate temperature control and calibration of the temperature sensor may be necessary $[4,5]$.

Manufacturing costs are high for previously proposed PCR microfluidic channel chips that used polydimethylsiloxane, polycarbonate, and poly(methyl methacrylate). In this paper, to reduce the manufacturing costs of the PCR chip, a study was carried out for the temperature measurement and calibration of a PCR chip fabricated by using a thin polychlorinated biphenyl(PCB) substrate, polymerfilm, and double-sided tape, respectively, for the chamber floor, ceiling, and walls.

Temperature is one of the most important factors in the PCR amplification process. Controlling the temperature of PCR amplification is accomplished by using the PCB's heating pattern and a negative-temperature-coefficient (NTC) thermistor sensor. When the temperature is effectively controlled, since a PCR can operate fast even in the same cycle and can be smaller in size, the mobility of the PCR will be more effective than that of

*Corresponding Author 
conventional devices.

The PCR chip studied in this paper uses an NTCthermistor, which has a $1 \%$ resistance error, as a temperature sensor. In this paper, PCR performance was compared for cases when the NTCthermistor was calibrated and not calibrated. The effect of thermistor calibration on PCR performance was investigated by comparing the band brightness of gel images obtained through electrophoresis for amplified DNA in both cases.

This paper introduces the materials and methods in Section 2 and presents the results in Section 3. The conclusion is made in Section 4.

\section{Materials and Methods}

\subsection{Micro PCR Chip}

The structure of the microPCR chip proposed in this study consists of four layers, as shown in Figure 1(a). When the experiment is carried out with only the PCB base, results are poor because DNA or fluorescent substances prone to be adsorbed. By attaching box tape (\#309 mini clear, 3M) made of PP (polypropylene) material on top, and 400$\mu$ mdouble-sided tape (5620A, Nitto) in the middle part of the chamber as chamber walls, a space inside the chamber was secured. The cover of the chamber was fabricated with a material using PP, which is a material of the tube actually used in a biology laboratory.

Figure 1(b) shows the bottom part of the PCB, which is on the opposite side of where the chamber will be located. To heat up the reagent, a heating pattern was constructed with copper wire. At the center of the heating pattern, an NTC thermistor sensor was attached for temperature measurement of the chamber. When the NTC thermistor sensor used in the chip measured temperature, the resistance value was measured and converted to the temperature using Steinhart-Hart $(\mathrm{SH})$ equation.

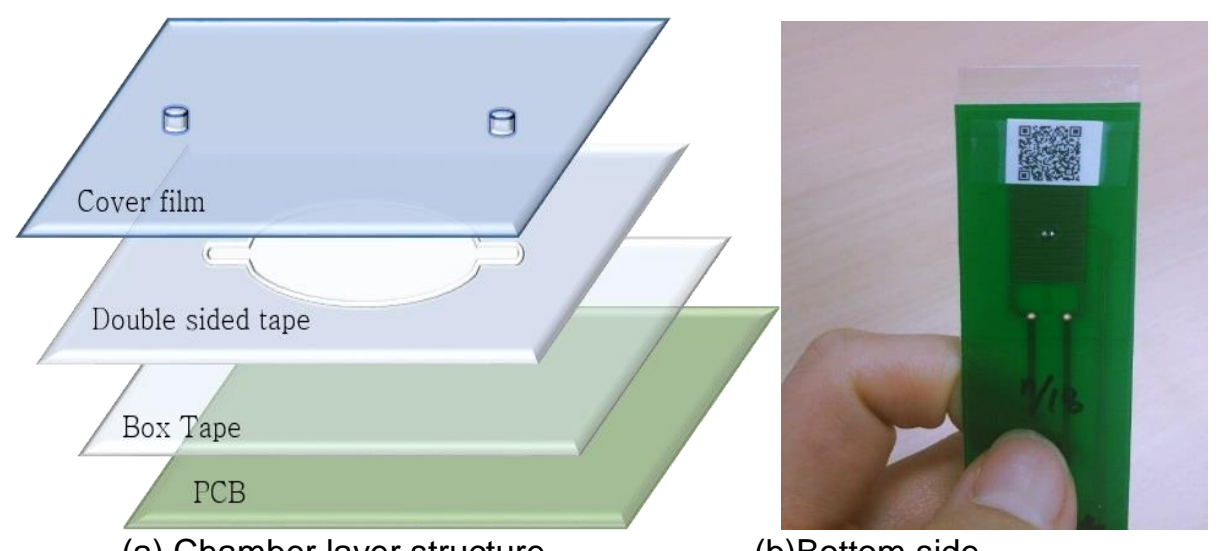

(a) Chamber layer structure

(b)Bottom side

Figure 1. Chip Structure

\subsection{NTC Thermistor}

The NTC thermistors employed in this study were chip thermistors with a resistance error of 1\% (NCP15XH103F03RC, DigiKey).Considering the possibility of lot variations among the thermistors, they were supplied by two companies (Mouser and DigiKey)and the thermistors from each company formed a group of thermistors. The previously supplied sensors from DigiKey with the same part number compose another group resulting in three groups of thermistors. For the experiment, the newly purchased thermistors from Mouser were marked as Group M, those purchased from DigiKey as Group D, and the previously purchased DigiKey thermistors as Group R. The number $n$ after each group's alphabetic designator indicates the $n$-th thermistor in its group.

Using the resistance values for four important temperatures $\left(50^{\circ} \mathrm{C}, 60^{\circ} \mathrm{C}, 70^{\circ} \mathrm{C}\right.$, and 
$95^{\circ} \mathrm{C}$ ) in the PCR from the temperature-resistance tables provided by the thermistor suppliers, the uncalibrated $\mathrm{SH}$ coefficients of $\mathrm{A}, \mathrm{B}$, and $\mathrm{C}$ were obtained. Using these values and the Steinhart-Hart formula $\left(\frac{1}{\mathrm{~T}}=A+B \ln (\mathrm{R})+\mathrm{C}(\ln (R))^{3}\right)$, the uncalibrated temperature values were calculated. The calibrated $\mathrm{SH}$ coefficients were determined by measuring the resistance values for each of four temperatures in a constanttemperature water tank. The calibrated temperature values were obtained by these coefficients.

In the PCR, the temperatures of three processes-denaturation, annealing, and extension-are important. In the current experiment protocol, the temperatures corresponding to the three stages are $95^{\circ} \mathrm{C}, 60^{\circ} \mathrm{C}$ (or sometimes $50^{\circ} \mathrm{C}$ ), and $72^{\circ} \mathrm{C}$. At $95^{\circ} \mathrm{C}$, eight chips that had thermistors showing the temperature values corresponding to the overall average value, and four chips showing maximum values and minimum values, were selected, for a total of 16 chips (Table 1).

The selected thermistors were divided into four groups, as shown in Table 2, considering that there were four units of micro PCR systems used in the experiment. Each group consisted of one chip showing a maximum value, one chip showing a minimum value, and two chips showing the values corresponding to the average value. For each group, the PCR tests were carried out in four systems simultaneously. As shown in Table 3 , four rounds of experiments were conducted. Throughout each round, the maximumvalue and minimum-value chips were mounted once each in the four systems, randomizing the system variation. In the table, MAX, MIN, and AVG are the chips showing maximum value, minimum value, and average value, respectively. In each round of experiments, two experiments were carried out using the uncalibrated and the calibrated coefficient alternatively.

Table 1. Maximum, Minimum, and Average Values at a Temperature of $95^{\circ} \mathrm{C}$, and the Corresponding Chip Numbers of the Three Groups

\begin{tabular}{|c|c|c|}
\hline & Temperature $\left({ }^{\circ} \mathrm{C}\right)$ & $\begin{array}{c}\text { Corresponding sensor chip numbers } \\
\text { of the three groups }\end{array}$ \\
\hline \multirow{3}{*}{ MAX } & 98.26 & D1 \\
\cline { 2 - 3 } & 96.77 & M29, M73 \\
\cline { 2 - 3 } & 96.42 & M27 \\
\hline \multirow{3}{*}{ MIN } & 94.50 & D17 \\
\cline { 2 - 3 } & 94.57 & R69 \\
\cline { 2 - 3 } & 94.73 & M6,M22 \\
\hline \multirow{2}{*}{ AVG } & 95.01 & D5,D21,D61,D85,R21,R29 \\
\cline { 2 - 3 } & 95.02 & R3,M9 \\
\hline
\end{tabular}

Table 2. Sixteen Chips Divided into Four Groups

\begin{tabular}{|c|c|c|c|c|}
\hline & Group 1 & Group 2 & Group 3 & Group 4 \\
\hline MAX & D1 & M29 & M73 & M27 \\
\hline MIN & M6 & M22 & R69 & D17 \\
\hline AVG & D5 & D21 & D61 & D85 \\
\hline AVG & R21 & R29 & R3 & M9 \\
\hline
\end{tabular}


Table 3. Arrangement of Thermistors for each Round of Experiments

\begin{tabular}{|l|c|c|c|c|}
\hline & Group 1 & Group 2 & Group 3 & Group 4 \\
\hline PCR_4 & MAX & AVG & AVG & MIN \\
\hline PCR_3 & MIN & MAX & AVG & AVG \\
\hline PCR_2 & AVG & MIN & MAX & AVG \\
\hline PCR_1 & AVG & AVG & MIN & MAX \\
\hline
\end{tabular}

\subsection{Micro PCR Device}

As shown in Figure 2, a PC, which is a host system, read the temperature values from the local system and calculated pulse width modulation (PWM) values on the basis of the proportional plus integral plus derivative (PID) control mechanism. In other words, the graphical user interface (GUI) was processed for the hardware task command and device status; and the micro PCR hardware, which is alocal system, performed hardware control including ADC and PWM with the PIC18F4550 microcontroller. A universal serial bus (USB), which is a standard link, was used to connect the host and local systems.

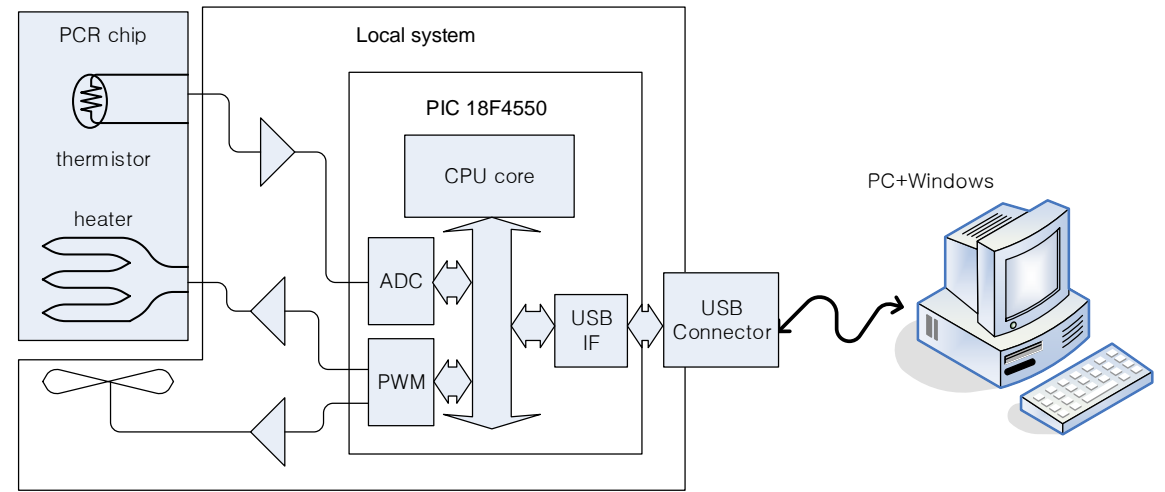

Figure 2. PCR Chip and Control System

With the four micro PCR systems configured as shown in Figure 3, two PCR experiments were carried out: one using the uncalibrated SH coefficients for 12 chips, and the other using the calibrated ones which were in the quick-response $(\mathrm{QR})$ codes. In a PCR chip, as shown in Figure 1(b), the calibrated SH coefficients were converted to a QRcode; and when the PCR chip was mounted on the micro PCR system, the QRcode was read and the calibrated SH coefficients were applied to the system. In the mean while, the uncalibrated coefficients, which could be pre-calculated from the resistancetemperature table provided by the manufacturer.

Since the PCR results according to the four micro PCR devices may be different from each other, a total of two sets were carried out. A set was defined as carrying out the experiment from Group 1 to Group 4, in which the chips corresponding to the maximum, minimum, and average values were selected on the basis of not being calibrated at $95^{\circ} \mathrm{C}$. This is shown in Table 3. Therefore, eight sets of results were produced in total: four sets for calibrated groups and four sets for uncalibrated groups. 


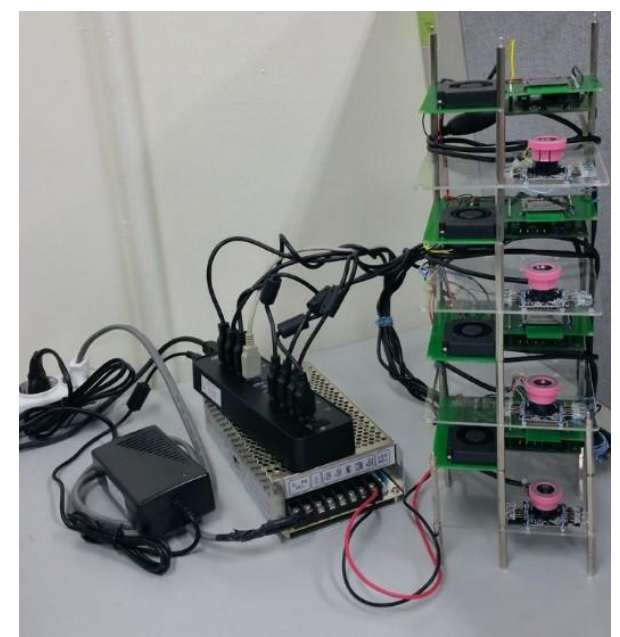

Figure 3. Four Units of the Micro PCR System Device

\subsection{Protocol and Reagent}

In this paper, the protocol was as follows: $3 \mathrm{~min}$ at $95^{\circ} \mathrm{C}$ for pre-heating; 39 cycles of 10 sat $95^{\circ} \mathrm{C}, 30$ sat $60^{\circ} \mathrm{C}$, and 30 sat $72^{\circ} \mathrm{C}$; and $3 \mathrm{~min}$ at $95^{\circ} \mathrm{C}$ and 30 sat $50^{\circ} \mathrm{C}$ for the final extension.

The followingmaterials were used in the experiment: $11 \mu \ell_{\text {of }} 1 \mathrm{ng} / 1 \mu \ell$ DNA (CT),

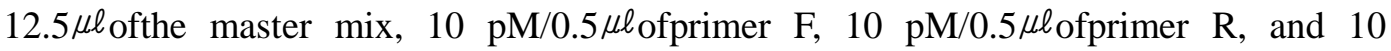
$\mathrm{pM} / 0.5 \mu \ell_{\text {ofthe probe. }}$

\subsection{Electrophoresisand UV Image Capturing}

The electrophoresis was carried out for $25 \mathrm{~min}$ at $135 \mathrm{~V}$ in the TBE $0.5 \mathrm{X}$ agarose gel. And to capture UV images, a Canon EOS 540Dcamera was used, and the images were captured by setting the f-stop at 5.6, ISOintensity at 1600, and focal distance at $42 \mathrm{~mm}$.

\section{Results}

Figure 4 shows the gel images for all four rounds of the experiments. The topmost gel image of the figure shows the first round of the experiment. The $1^{\text {st }}$ and the last columns of each round image are the ladders, and the $2^{\text {nd }}$ and $3^{\text {rd }}$ columns show the bands of the amplicons from the commercial thermal cycler. The even ones show the bands of the amplicons from the calibrated chips, and the odd ones show the bands of the uncalibrated chips. In the $3^{\text {rd }}$ round of experiments, it was found that the band for one of uncalibrated $\operatorname{chips}\left(7^{\text {th }}\right.$ column of the $3^{\text {rd }}$ image $)$ was not shown. With the naked eye, it was difficult to distinguish between the band brightness of the amplified amplicon in a calibrated chip and that of an uncalibrated chip. 


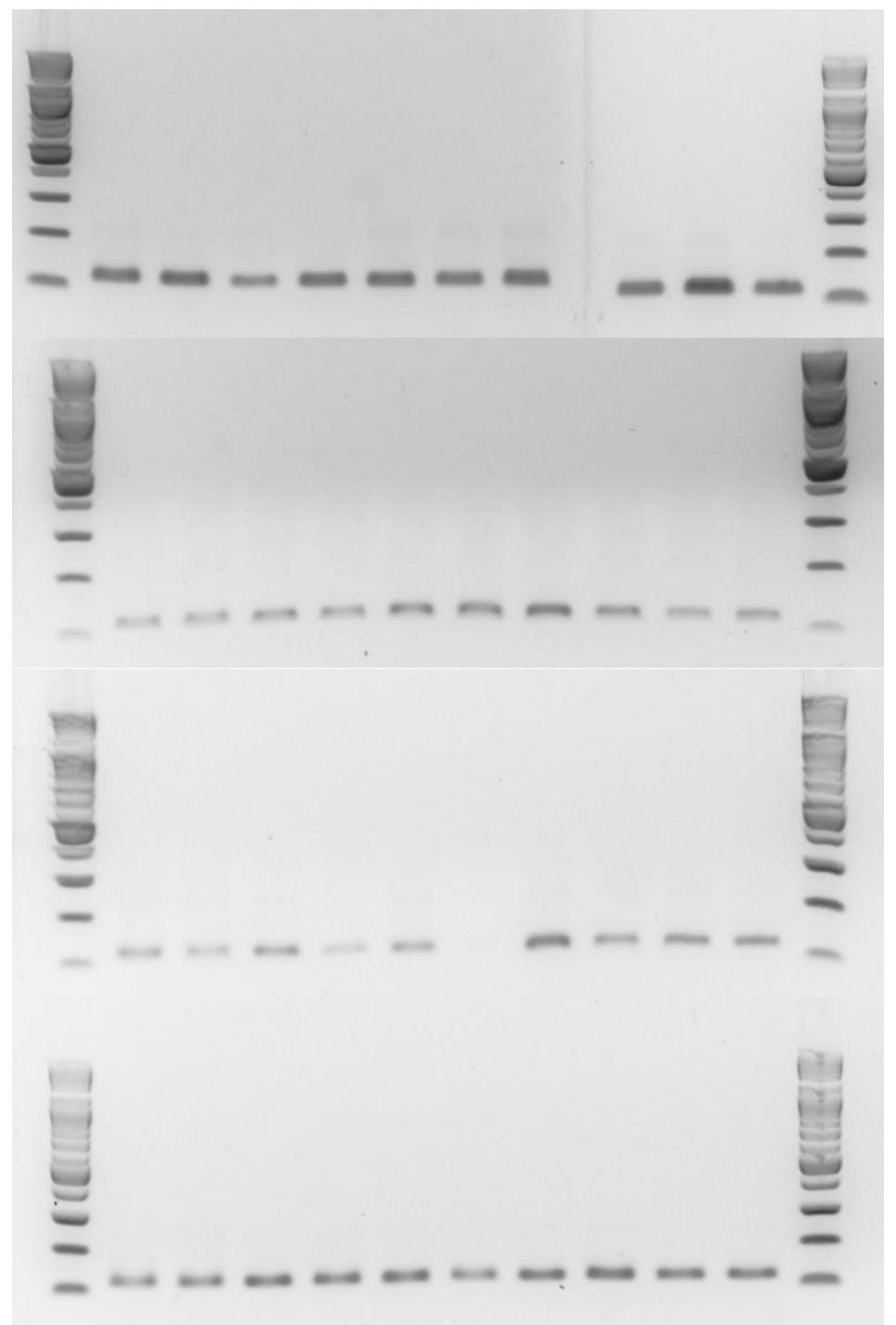

Figure 4. Gel Images for all Four Experimental Rounds. (The Topmost Gel Image Shows the Results of the First Round)

To quantitatively analyze the brightness of the bands, freeware called Gel Analyzer was used. As shown in the figure, because the background brightness changed vertically, the change in background was compensated for by using the rolling-ball algorithm before detecting a peak (the ball radius was 120 pixels). This example is shown in Figure 5. In the upper graph of the figure, the brightness profile of the lane located on the $8^{\text {th }}$ column in the $2^{\text {nd }}$ gel image was drawn by overlaying it on the trajectory of the rolling ball. In the lower lane image, the peak zone and ridge zone for calculating the band intensity volume are marked in red lines.

In Table 4, the intensity volumes of bands extracted as above were summarized. In the first row of the table, the column numbers are shown. From the second row on, the brightness volumes of the bands are summarized sequentially, starting from the top gel image. The first and last columns show the volumes of the last bands of the ladder. The $7^{\text {th }}$ column of row R3 was left blank because a band was not detected.

As shown in Figure 4, the ladder brightness of each gel image was different. In other words, even if the DNA amounts are the same, the band brightness will be different. To compensate for this, it was normalized with the volume of the last ladder band, which is the ladder band that is the most similar to the molecular weight of amplicons. This is 
shown in Table 5, where the bands of calibrated chips and the bands of uncalibrated chips from Table 4 were grouped and re-arranged. As shown in Table 5, the average brightness volumes of amplicons produced from the calibrated chips were slightly larger. This fact becomes clear when looking at the box plot shown in Figure 6.

However, as a result of the t-test or ranksum test, the band volumes from the calibrated chips were not significantly larger ( $\mathrm{t}$-test $\mathrm{p}$-value $=0.45$, ranksum test $\mathrm{p}$-value $=0.41$ ). There is no basis for assuming that significant results will be obtained if more experiments are conducted. However, based on the box-plot result for the case of measuring temperatures with the thermistor, it is expected that better results will be obtained through calibration.

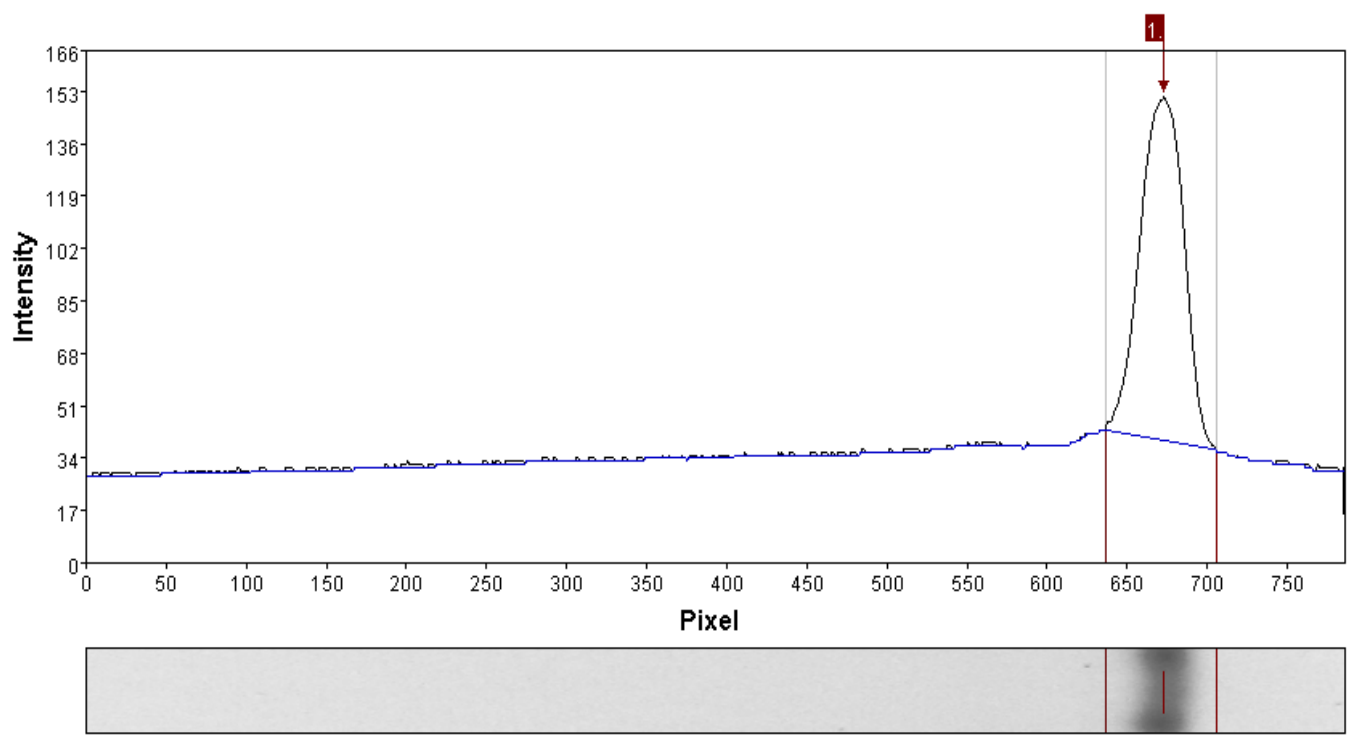

Figure 5. Band Detection Example using Gel Analyzer. Upper Graph: the Profile of the $8^{\text {th }}$ Column of the $2^{\text {nd }}$ Gel Image of Figure4.Lower Image: the Extracted Lane Image and Band Volume Calculation Area (Red Lines)

Table 4. Calculated Band Volume

\begin{tabular}{|l|r|r|r|r|r|c|c|c|c|c|c|c|}
\hline & \multicolumn{1}{|c|}{1} & \multicolumn{1}{|c|}{2} & 3 & 4 & 5 & 6 & 7 & 8 & 9 & 10 & 11 & \multicolumn{1}{c|}{12} \\
\hline R1 & 2313 & 3569 & 3798 & 2286 & 3497 & 3641 & 3157 & 3879 & 3731 & 5238 & 3737 & 2881 \\
\hline R2 & 437 & 995 & 1152 & 1586 & 1390 & 2132 & 2412 & 2911 & 1865 & 1142 & 1132 & 690 \\
\hline R3 & 644 & 1208 & 830 & 1640 & 565 & 1294 & & 3084 & 1599 & 1979 & 1947 & 1290 \\
\hline R4 & 2234 & 2168 & 2471 & 3075 & 2896 & 3086 & 2084 & 3041 & 3532 & 2833 & 2849 & 2642 \\
\hline
\end{tabular}

Table 5. Band Volumes Normalized by the Last band Volume of the Ladder

\begin{tabular}{|c|r|r|r|r|r|r|r|r|}
\hline & \multicolumn{3}{|c|}{ Band volumes for the calibrated chips } & \multicolumn{3}{|c|}{ Band volumes for the uncalibrated chips } \\
\hline R1 & 0.88 & 1.40 & 1.49 & 2.02 & 1.35 & 1.22 & 1.44 & 1.44 \\
\hline R2 & 2.81 & 3.78 & 5.17 & 2.03 & 2.47 & 4.28 & 3.31 & 2.01 \\
\hline R3 & 1.70 & 1.34 & 3.19 & 2.05 & 0.58 & 0.00 & 1.65 & 2.01 \\
\hline R4 & 1.26 & 1.27 & 1.25 & 1.16 & 1.19 & 0.85 & 1.45 & 1.17 \\
\hline mean & \multicolumn{3}{|c|}{2.05} & & \multicolumn{7}{c|}{1.65} \\
\hline std & \multicolumn{3}{|c|}{1.15} & & \multicolumn{7}{c|}{1.03} \\
\hline
\end{tabular}




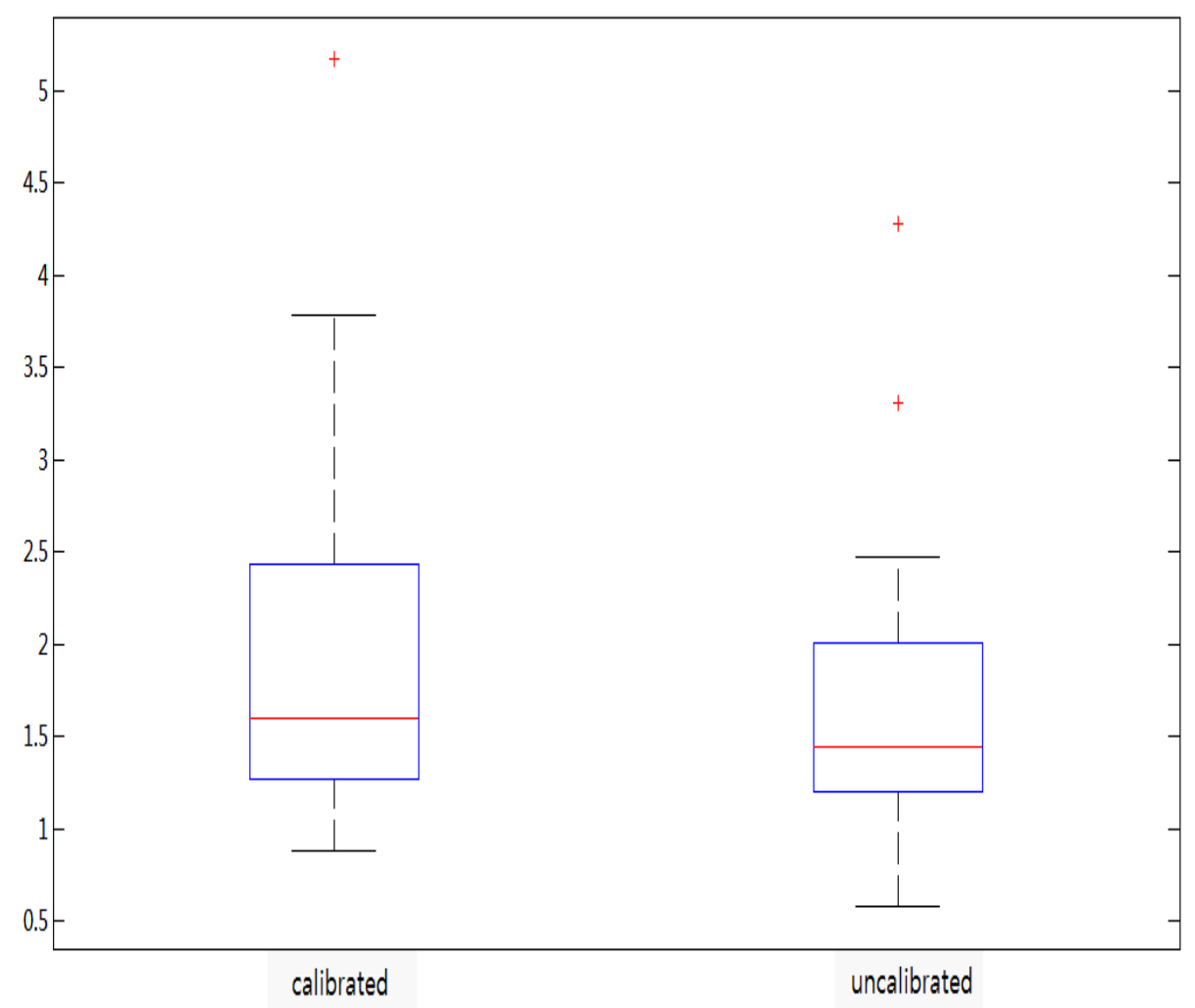

Figure 6. Box Plot of Band Volumes Shown in Table

\section{Conclusion}

In this study, the effect of thermistor calibration on the performance of a PCR was investigated by conducting experiments of the PCR process with chips that were calibrated and not calibrated with a NTC thermistor that has a $1 \%$ resistance error. This is required for measuring temperature in a micro PCR chip, which is a reaction chamber constituted on a thin PCB substrate.

Using the Steinhart-Hart formula, eight chips in which a thermistor showed a temperature value corresponding to the overall average value at $95^{\circ} \mathrm{C}$, and four chips showing maximum and minimum values, were selected for a total of 16chips. Using four units of the micro PCR as local systems, experiments were carried out.

The average brightness volume (1.15) of amplicons produced from the calibrated chips using GelAnalyzer was slightly larger than the average brightness volume (1.03) of amplicons produced from the uncalibrated chips. This was more apparent in abox plot. However, as a result of a t-test or ranksum test, the band volume from the calibrated chips was not significantly large ( $\mathrm{t}$-test $\mathrm{p}$-value $=0.45$, rank test $\mathrm{p}$-value $=0.41$ ).

Although significant results were not obtained, as a result of the box plot, it is expected that better results will be obtained through calibration when the temperature is measured with a thermistor. 


\section{Acknowledgements}

This research was supported by the "Research and Business Development, 2013" project of the Ministry of Trade, Industry and Energy (N0000907) and Basic Science Research Program through the National Research Foundation of Korea(NRF) funded by the Ministry of Education (2013R1A1A2013490).

\section{References}

[1] D. Patkoa, Z. Martonfalvic, B. Kovacsa, F. Vonderviszta, M. Kellermayerc, and R. Horvatha, "Sensors and Actuators", vol. 196, no. 352, (2014).

[2] M. Focke, D. Kosse, C. Muller, H. Reinecke, R. Zengerle, and F. Stettenab, "Lab on a Chip 10", no. 1365, (2010).

[3] S. Haeberle and R.Zengerle, "Lab on a Chip 7", no. 1094, (2007).

[4] C. Koo, M. Malapi-Wight., H. S. Kim, O.S. Cifci, V. L. Vaughn-Diaz, B. Ma, and A. Han, "PloSONE", vol. 8, no. 82704, (2013).

[5] E. Salm, Y.-S. Liu, D. Marchwiany, D. Morisette, Y. He, L. Razouk, A. K. Bhunia, R. Bashir, "Biomedical Microdevices", vol. 13, no. 1095, (2011).

[6] P. S Dittrich and A. Manz, "Nature Reviews Drug Discovery", vol. 5, no. 210, (2006).

[7] A.M. Foudeh., T.F. Didar, T. Veres, and M. Tabrizian, "Lab on a Chip 12", no. 3249 (2012).

[8] K.K. Kumar, R.P. Jayaraman, and S.K. Narasimhal, "Hand held micro PCR device", (2008)

[9] M. M. Hoehl, M. Weißert, A. Dannenberg, T. Nesch, N. Paust, F. von Stetten, and J. Stei-gert, "Biomedical Microdevices", vol. 16, no. 375, (2014).

[10] H.B. de Torres, C.Rensch, M. Fischer, and A. Schober, "Sensors and Actuators", A 160, 109, (2010).

[11] J.W. Howes, "Journal of the British Institution of Radio Engineers", vol. 13, no. 228, (1953).

\section{Authors}

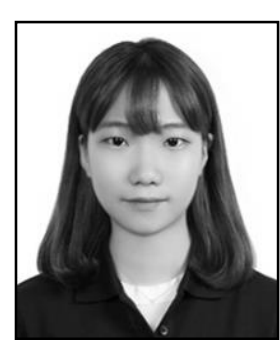

Seul-Bit-Na Koo, she received the B.S. degree in Ubiquitous Computing from Hallym University.She is enrolled in a master degree in Convergence Software in Hallym University..She recent interests focus on biomedical system and embedded system.

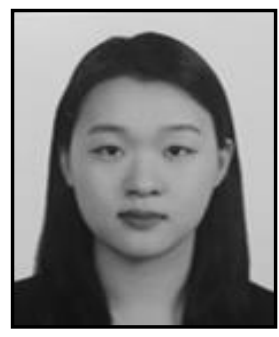

Cho-Jae Hyeon, she currently studies in a Bachelor and Master degree in Convergence Software in Hallym University.She recent interests focus on biomedical system and embedded system.

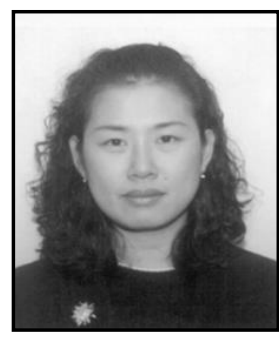

Hye-Jeong Song, she received the Ph.D. degree in Computer Engineering from Hallym University. She is a Professor in Department of Convergence Software of Hallym University, Korea. His recent interests focus on biomedical system and bioinformatics. 


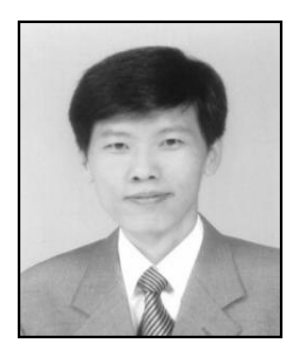

Chan-Young Park, he received the B.S. and the M.S. from Seoul National University and the Ph.D. degree from Korea Advanced Institute of Science and Technology in 1995. From 1991 to 1999, he worked at Samsung Electronics. He is currently a Professor in the Department of Convergence Software of Hallym University, Korea. His research interests are in Bio-IT convergence and sensor networks.

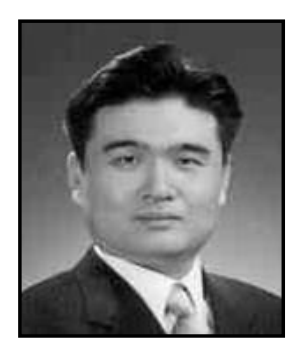

Yu-Seop Kim, he received the Ph.D. degree in Computer Engineering from Seoul National University. He is currently a Professor in the Department of Convergence SoftwareofHallym University, Korea. His research interests are in the areas of bioinformatics, computational intelligence and natural language processing.

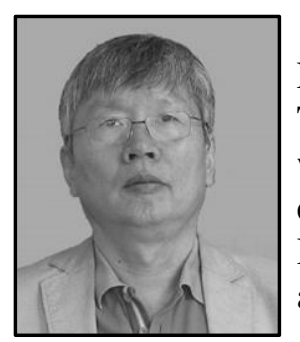

Jong-Dae Kim, he received the M.S. and the Ph.D. degrees in Electrical Engineering from Korea Advanced Institute of Science and Technology, Seoul, Korea, in 1984 and 1990, respectively. He worked for Samsung Electronics from 1988 to 2000 as an electrical engineer. He is a Professor in Department of Convergence Software, Hallym University. His recent interests focus on biomedical system and bioinformatics. 